\title{
IVRS BASED NEWS EXTRACTING SYSTEM
}

\author{
Ritesh Sharma ${ }^{1}$, Niteesh Dubey ${ }^{2}$, Naresh Thoutam ${ }^{3}$, Shoyeb Pathan ${ }^{4}$ \\ ${ }^{I}$ Department of Computer Engineering, Sandip Institute of Technology \& Research Centre \\ ${ }^{2}$ Department of Computer Engineering, Sandip Institute of Technology \& Research Centre \\ ${ }^{3}$ Department of Computer Engineering, Sandip Institute of Technology \& Research Centre \\ ${ }^{4}$ Department of Computer Engineering, Sandip Institute of Technology \& Research Centre
}

\begin{abstract}
In today's era, the human being is very concern about to know its circumstances behavior whether it is environmental, politics, current affairs, sports or the weather condition of upcoming days i.e. News, which can be easily available through television or internet sources in our daily routine but it's one of the human tendency, to feel the peak level of necessity of the something when we know to acquire such facility in particular situation is truly impossible to deal with such occurrences, our system can fulfill their needs just by dialing one toll free number through user i.e. 1800-XX-XXXX, where our smart and intelligent IVRS based news extractor will take care of their needs and fulfils it by responding in voice based form. Best part of this system is user himself will not be able to compare that in spite of any human operator , a genius system is responding to his needs. Proposed system will be able to automatically answer the user's call and can play the welcome tone that will be the pre-recorded voice and allow user to select his news domain. It will acquire the input as a $D T M F$ signals through users cell phone's keystrokes after analyzing the signals meaning it will extract the related news information from data source. System will provide the main categories and sub-categories to fulfill the user's requirements optimally, motive of designing such smart system is to accomplish the society needs with modern technical assistance and make people's life easier and convenient.
\end{abstract}

Keywords-DTMF, IVRS, Speech Synthesis

\section{INTRODUCTION}

Consider the situations like, any user on his business tour and travelling from Bangalore to Mumbai via train and somehow he was unable to subscribe the internet service in his cell phone, and interested to know rain fall status of Mumbai or a blind but responsible \& patriotic citizen of an India is very eager to know about results of prime minister's election poles or a farmer wishes that anyhow he can know the weather information of upcoming days so that he can plan its agricultural activities.

In above all three cases user is eagerly waiting to acquire his requirement but due to some circumstances he is unable to get that, like for person on its business tour is able to see and read the information but he is not having such source in his journey and person is in second case is although well qualified and concern but due to its blindness, he can't read the newspaper or operate news channels on televisions efficiently ,in third case although the poor farmer is able to see but not that much qualified to read and acquire its need through the news channels or weather journals. So in such scenario traditionally user will wait, wait and wait only and at last he will prepare himself for facing worst situation but in era of modern science, our proposed system will act as magic wands for them. In our system all users can fulfill their needs just by dialing one toll free number ex. 1800-XX-XXXX, where our smart and intelligent IVRS based news extractor will take care of their needs and fulfills it by responding in voice based form.

Problem Definition: To Design a system which is able to provide voice based response to user as per his/her needs via accepting the input as DTMF touchtone.

\section{REQUIREMENTS AND CHALLENGES}

It is observed that accepting natural language as a input for any system enhance the ease of accessibility for any user to satisfy his/her needs ,but it is also true that it is not too easy to implement voice synthesis units for all local languages [4]. Accepting standard integers as a input provide any system a great ease, hence IVRS uses numeric inputs for accepting selection through users [4,3]. Managing CSR resource for any organization lead another overhead for them where as IVRS system overcome those issues via replacing manual intervention through automation of functionality of serving users[1].

\section{PROPOSED SYSTEM}

\subsection{Interactive Voice Response (IVR) System}

It is a communication system which provides automated telephone call access to specified computer database information. Interactive Voice Response is a methodology that lead computer to detect Dual tone multi frequency key inputs and produces 
voice response as per the input. Dual tone multi frequency signaling allows for telecommunication signaling over analog telephone lines in the voice-frequency band between telephone/cell phone equipment and other communications devices.

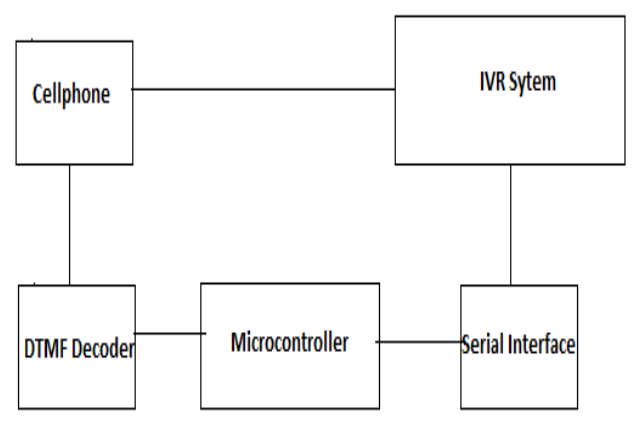

Fig 1 IVRS Basic Block

\subsection{DTMF (Dual Tone Multi Frequency)}

It is the frequency to the phone companies that we produce when we press telephone's keys. it's also known as Touchtone. With DTMF, each key you press on your phone generates two tones of specific frequencies. So that a voice can't imitate the tones, one tone is generated from a high- frequency band of tones and the other from a low frequency band.

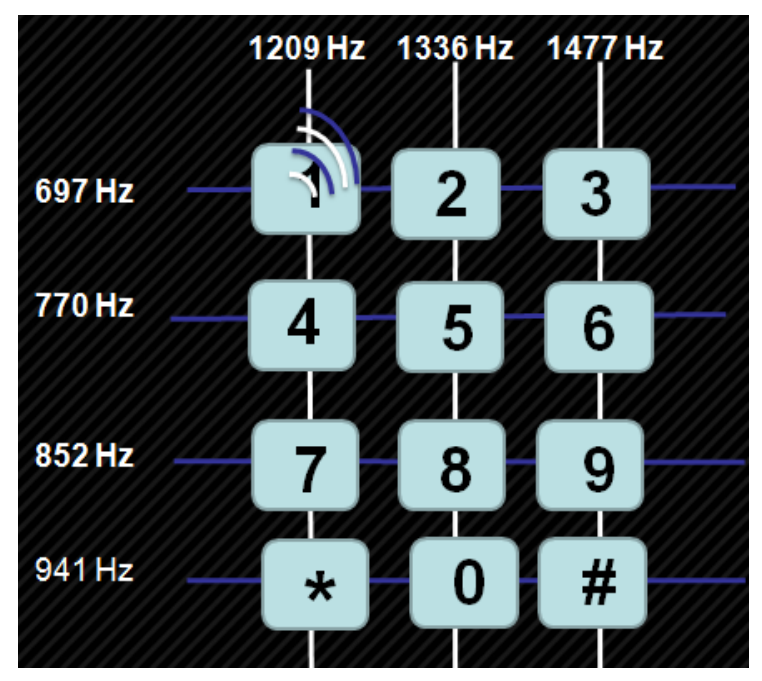

Fig 2 DTMF Generation

\subsection{Speech Synthesis}

Speech synthesis is a process of artificially produced human speech. A system used for this cause is called a speech synthesizer. A text-to-speech (TTS) system converts natural language text into speech.

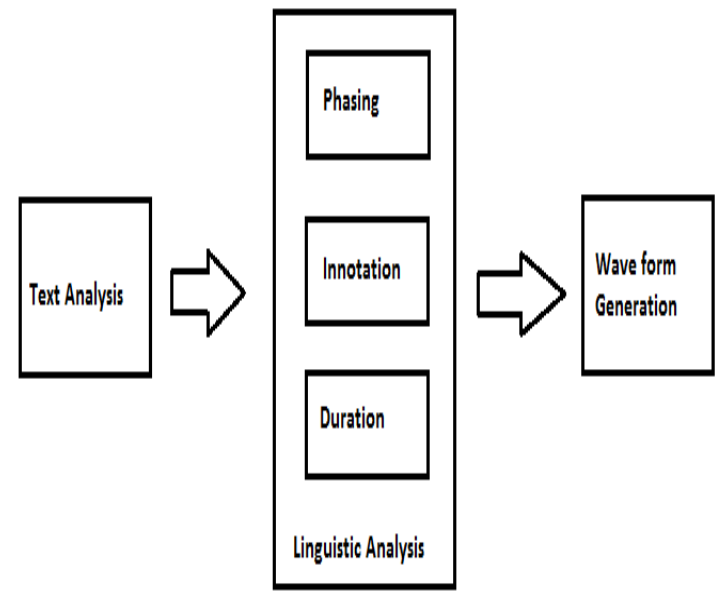

Fig 3 Text To Speech Conversions

\section{SYSTEM DESIGN}

Proposed System receives calls automatically from user and it will provide welcome tone, News domain menu to the user for further selection which will be made by user as per his/her needs ,once the news domain is decided system will respond to user for making selection on sub domain of news after accepting selection on sub categories of news system will acquire the particular specified news data from news database and deliver it to text to speech module for processing text data and transforming it into voice based form. Transformed voice based news will delivered to the user.

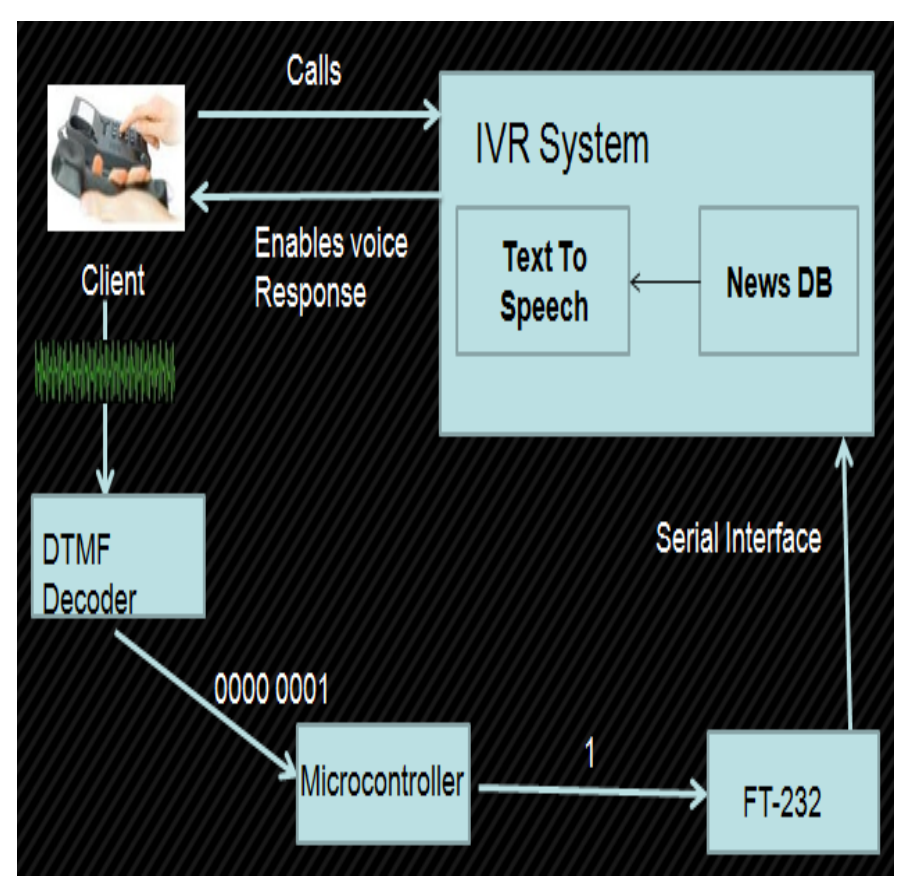

Fig 4 System Architectural view 


\section{MATHEMATICAL MODEL}

System is depicted as in various phases to elaborate system's behavior mathematically.

\section{Phase: 1}

Caller would be the entity whose unique identification would makes the service secure and reliable i.e. $\mathrm{C}=$ $\{\mathrm{c} 1, \mathrm{c} 2, \mathrm{c} 3 \ldots \ldots \ldots . . . . \mathrm{cn}\}$ Where , $\mathrm{C}[\mathrm{i}] \leftarrow$ customer unique identification no. i.e. 91-8655123123, News resided in database is only the need and feature of whole system that can be represented as, $\mathrm{W}=\{\mathrm{w} 1, \mathrm{w} 2, \mathrm{w} 3 \ldots . . . \mathrm{wn}\}$ Where, $\mathrm{W}[\mathrm{i}] \leftarrow$ News related to specific domain. Each caller will press the keypad for making the precise selection as per his need each pressed keypad will induce the unique analog DTMF frequency i.e. $=\{a 0$, a1,a2......a12 \} Where, A[ i ] $\leftarrow$ Analog signal for keypad [ i ].

Each induced analog signal should be get rectified in its readable digital form through DTMF reader i.e. DT $=$ DTMF Decoder, Each analog signal will be get process through the DTMF decoder FT8870 and will output the digital BCD(Binary Coded Decimal) for further processing i.e. $\mathrm{B}=$ $\{\mathrm{b} 1, \mathrm{~b} 2, \mathrm{~b} 3 \ldots . . . . . . \mathrm{bn}\}$ Where, $\mathrm{B}[\mathrm{i}] \leftarrow \mathrm{BCD}$ for specific analog frequency $\mathrm{A}[\mathrm{i}$ ] . Binary coded decimal values will be bring as input to the Atmega8 microcontroller for further processing ,which will convert the BCD into equivalent decimal digits. $\mu \mathrm{c}$ $=$ Microcontroller.

$\mathrm{D}=\{\mathrm{d} 0, \mathrm{~d} 1, \mathrm{~d} 2 \ldots \ldots . . . \mathrm{dn}\}$ Where'd $[\mathrm{i}] \leftarrow$ Decimal for specific $\mathrm{BCD}, \mu \mathrm{c}(\mathrm{B}[\mathrm{i}])$ ).Acquired d[i] through $\mu \mathrm{c}$, will be transmitted to the system which would be responsible for matching the input data with repository via serial bus i.e. Sbus .System will possess the data repository unit which will maintain the whole records, $\log$, reports etc. $\mathrm{P}=\{\mathrm{c} 1 \mathrm{U}$ a1 $\mathrm{U}$ w1, c2 $\mathrm{U}$ a2 $\mathrm{U}$ w2,........, cn $\mathrm{U}$ an $\mathrm{U} w \mathrm{w}\}$, Selected or extracted result will be acknowledged to the caller in the voice based form

$\mathrm{V}=\{\mathrm{v} 1, \mathrm{v} 2, \mathrm{v}\}$ ,vn $\}$ Where, V [ i ] $\leftarrow$ Voice response towards the customer for individual selection $\mathrm{A}[\mathrm{i}]$.

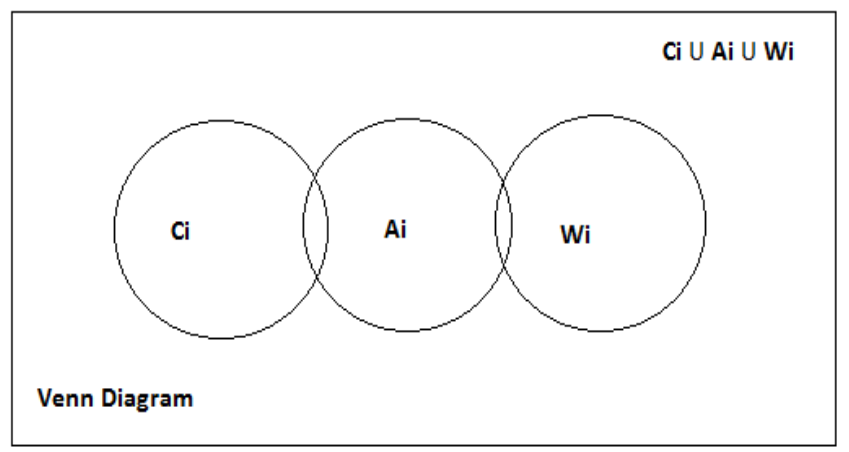

Fig 5 Data repository Venn Representation

\section{Phase: 2}

As soon as the caller will be get automatically attended by the IVRS actual procedure will carry out in following manner, If (Auto-answering)

Then

$$
\begin{aligned}
& P n \leftarrow C n ; \\
& C n \leftarrow V n ; \\
& D \leftarrow A[i] ; \\
& B \leftarrow D ; \\
& \text { Phase } 3() ;
\end{aligned}
$$

Else

$$
\text { Busy Mode ( ); }
$$

Kill Phase ( );

The above assignment shows that once the auto-answering is performed caller information (91-8655123123) would be transferred to the data repository unit, where in response IVRS will throw the voice based welcome tone to the caller as a ack of call and also provide the set of domain options respectively and as soon as an user will select the domain through key press, individual DTMF signals will be transmitted to the MT8870 decoder then decoder will generate the $\mathrm{BCD}$ code for the acquired analog signal and phase-3 will initiate.

\section{Phase: 3}

If (B)

Then

\section{Generate Exception \\ Kill Phase ( );}

As soon as the BCD code will get generated each BCD code will get transfer to the microcontroller and then it will generate the decimal number for further processing, after that microcontroller will transmit that decimal number towards IVRS through serial bus interface

\section{Phase: 4}

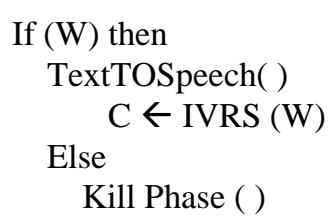

In above phase, it shown that once the required news is fetched from repository performs voice synthesis for it and transmits it to the caller. 


\section{Phase: Kill Phase}

AbnormallyTerminationOfCall ( );

This phase will just terminate the session of the current user during invalid circumstances.

\section{CONCLUSIONS}

Specified IVRS based news extracting system is capable of providing the user required news information through voice based response which also make difference to their knowledge base and needs, irrespective of any eligibility like literacy, qualification, physical fitness for that user just need to make some selections and can make their life easier and convenient.

\section{ACKNOWLEDGEMENTS}

We are thankful to our Principal Dr. S.T. Gandhe, whose time to time help and valuable guidance gave us high moral support. We would also like to sincerely thank our coordinator of Computer Department Prof.Sandip Walunj for his guidance and constant encouragement. We are also grateful to Prof.Naresh Thotam and Prof Leena Deshmukh for their encouraging guidance; we are highly obliged to the entire staff of the Computer department for their kind cooperation and help.

\section{REFERENCES}

[1] Kosuke Hashizumea, Tuan Phung-Ducb, Shoji Kasaharaa and Yutaka Takahashia "Queueing Analysis of Internet-Based Call Centers with Interactive Voice Response and Redial." $17^{\text {th }}$ IEEE. CAMAD, 2012.

[2] Noah Gans, Ger Koole, Avishai Mandelbaum "Telephone Call Centers: Tutorial, Review, and Research Prospects." SOM's Project, March 17, 2003.

[3] Ms. Seema P. Mishra, Swapnil S. Gourkar "Interactive Voice Response System For Educational Institution." International Journal of Advanced Engineering Technology, E-ISSN 0976-3945.

[4] Aditi Sharma Grover, Osamuyimen Stewart, David Lubensky, "Designing Interactive Voice Responses (IVR) Interfaces: Localization for Low Literacy Users", Meraka Institute, CSIR and IBM Research.

[5] Future Technology Devices International ltd "FT232BM USB UART IC." Datasheet Version 2.0 , Document No.: FT_000206 FT232BM , Clearance No.: FTDI\# 133, 21st September 2010.

[6] Zarlink Semiconductor "MT8870/MT8870-1 ISO2-CMOS Integrated DTMF Receiver.” May 2005.

[7] R. Srinivasan, J. Talim, and J. Wang, "Performance analysis of a call center with interactive voice response units," Top, vol. 12, no. 1, pp. 91-110,2004.

[8] J. Sherwani, N. Ali, S. Mirza, A. Fatma, Y. Memon, M.Karim, R. Tongia \& R. Rosenfeld, HealthLine: Speech-basedAccess to Health Information by
Low-literate Users, Proc.IEEE/ACM International Conference on Information andCommunication Technologies and Development(ICTD2007), India, 2007.

[9] A. Kumar, N. Rajput, D. Chakraborty, S. Agarwal \& A.A. Nanavati, WWTW: The World Wide Telecom Web,Proc. 2007 Workshop on Networked Systems for DevelopingRegions (NSDR 07), Japan, 2007. 